\title{
An Analysis of the Putative CBD Binding Site in the lonotropic Cannabinoid Receptors
}

\author{
Chanté Muller and Patricia H. Reggio* \\ Department of Chemistry and Biochemistry, University of North Carolina at Greensboro, Greensboro, NC, United States
}

Cannabinoids have been long studied for their therapeutic properties, particularly for their use in the treatment of pain. As new therapies are sought after to treat conditions of chronic pain, so is a better understanding of the ligands and their target receptors or channels. A recently published cryo-EM structure showed the putative binding location of a well-known cannabinoid ligand, cannabidiol (CBD), in TRPV2, a channel that has been implicated in inflammation and chronic pain. TRPV2, along with TRPV1, TRPV3, TRPV4, TRPA1, and TRPM8 all have the capability to be modulated by cannabinoid ligands and are located in the peripheral nervous system. Here, we analyze the putative CBD binding site in each of these channels and compare structural and sequential information with experimental data.

OPEN ACCESS

Edited by:

Senthilkumar Rajagopal, Rayalaseema University, India

Reviewed by:

Saravanan Ramachandran, Chettinad University, India Prasanna K. Santhekadur, JSS Academy of Higher Education and Research, India

*Correspondence: Patricia H. Reggio phreggio@uncg.edu

Specialty section:

This article was submitted to Cellular Neurophysiology, a section of the journal Frontiers in Cellular Neuroscience

Received: 10 October 2020 Accepted: 18 November 2020 Published: 09 December 2020

Citation: Muller C and Reggio PH (2020) An Analysis of the Putative CBD Binding Site in the lonotropic Cannabinoid Receptors. Front. Cell. Neurosci. 14:615811. doi: 10.3389/fncel.2020.615811
Keywords: cannabidiol, TRP channels, TRPV1, TRPV2, TRPV3, TRPV4, TRPA1, TRPM8

\section{INTRODUCTION}

Transient receptor potential (TRP) ion channels are membrane-spanning channels that are formed by the homo- or hetero-tetramerization of TRP subunits. Each subunit contains six transmembrane helices (S1-S6), which, when tetramerized together, form a central pore, allowing for cation permeation (Levine and Alessandri-Haber, 2007). These channels, located in the plasma membrane, are capable of gating several mono- and di-valent cations through this pore in response to a stimulus. In mammals, six main subfamilies of TRP channels have been identified: ankyrin (TRPA), vanilloid (TRPV), melastatin (TRPM), canonical (TRPC), mucolipin (TRPML), and polycystin (TRPP) (Winter et al., 2013). Several of these channels have been implicated as sensors of many pathological and physiological processes including itch, temperature, genetic disorders, and pain related to cancers, AIDS, or other neuropathic conditions (Nilius and Vennekens, 2007; Vay et al., 2012; Perálvarez-Marín et al., 2013).

Chronic pain conditions remain a significant and prominent problem in today's society, effecting millions of people worldwide (Starkus et al., 2019). The complex mechanisms and etiologies that underlie chronic pain are diverse and cover a range of symptoms, conditions, and pathways (Levine and Alessandri-Haber, 2007) that can be brought on by a variety of causes including diabetes (Szallasi et al., 2007), stroke (Lau and Vaughan, 2014), and treatments for other conditions. Often, other symptoms like depression, anxiety, fatigue, and limitation of activity co-occur leading to an overall reduced quality of life (Ware et al., 2002). The current therapies to treat chronic pain conditions are considered to be relatively inadequate. NSAIDs, opioids, tricyclic antidepressants, local anesthetics, and antiepileptics can work to alleviate some chronic pain sufferers' experiences but don't often produce sustained relief (Levine and Alessandri-Haber, 2007; Luongo et al., 2017). With opioid medications, there are also dangers of dependence, tolerance, and addictive behaviors 
associated with their usage. In an effort to combat the overuse of opioid medications and resultant side effects, as well as find other meaningful therapies, different avenues of pain-related therapeutics are being investigated, notably the use of Cannabis in the treatment of chronic pain (Nielsen et al., 2017).

This perspective aims to discuss the putative binding site of a well-known cannabinoid, cannabidiol (CBD) in a selection of six TRP channels that are located in primary somatosensory neurons. This selection of channels (TRPV1-TRPV4, TRPA1, and TRPM8) have been identified as thermoTRPs, responding to various thresholds of temperature activation, as well as ionotropic cannabinoid receptors due to their ability to be modulated by cannabinoid ligands (Muller et al., 2019). The putative binding site of CBD has been identified in TRPV2 (Pumroy et al., 2019), and in an effort to better understand CBD interaction at the ionotropic cannabinoid receptors, a sequential and structural analysis will be discussed herein.

\section{CANNABINOIDS AND THE MODULATION OF PAIN}

Cannabis has been used for millennia to treat pain caused by various situations, including uses in ameliorating pain caused by childbirth in ancient Israel, as a surgical anesthetic in China, and for various painful ailments in the West in the 1800s (Walker and Huang, 2002; Ware et al., 2002). Today, one of the most commonly cited reasons for seeking medical marijuana is due to chronic pain (Ilgen et al., 2013; Klimkiewicz and Jasinska, 2018). There is extensive literature that supports the role of phytogenic and endogenous cannabinoid ligands as pain modulators (Maione et al., 2006; Lau and Vaughan, 2014), and the identification of broader targets for cannabinoid ligands also works to support this hypothesis (Caterina, 2014). Canonically, CB1 and CB2 are most widely known as receptors for cannabinoid ligands (Howlett, 2002; Pertwee et al., 2010; Morales et al., 2018). Pharmacological evidence shows, however, that other G protein-coupled receptors (GPCRs) (Morales et al., 2018) and other receptor types, including a subset of TRP channels, also have the ability to be modulated by cannabinoid ligands. Of the subfamilies of TRP channels found in mammals, at least three subfamilies contain channels that have been identified as having this ability, earning the name "ionotropic cannabinoid receptors" (Jordt et al., 2004; Akopian et al., 2009). Additionally, these channels, TRPV1-4, TRPA1, and TRPM8 can be found in primary somatosensory neurons, acting as sensory transducers that may participate in the generation of painful sensations evoked by thermal, mechanical, or chemical stimuli. These attributes make the ionotropic cannabinoid receptors a worthwhile target to investigate for the development of new pain therapies by targeting that which contributes to the detection of stimuli.

\section{LOCATION, LOCATION, LOCATION}

The location of the TRP channels assessed in this perspective covers many neuron types within the peripheral nervous system, ranging from small diameter peripheral sensory nerves (TRPM8) (Peier et al., 2002b), to small- to medium-diameter neurons (TRPV1) (Caterina et al., 1997; Tominaga et al., 1998; Sanchez et al., 2001), to medium- to large-diameter neurons that give rise to $A \delta$ and $A \beta$ fibers (TRPV2) (Caterina et al., 1999; Bender et al., 2005), to co-expression with TRPV1 (Story et al., 2003) in a subset of small- to medium-diameter neurons (TRPA1) (Bautista et al., 2005; Kobayashi et al., 2005; Caterina, 2006), and to predominant expression in keratinocytes (TRPV3 and TRPV4) (Peier et al., 2002a; Mandadi et al., 2009). These channels (TRPV1-4, TRPA1, and TRPM8), having a wide distribution throughout the peripheral nervous system, have been implicated in roles of conducting various sensations, some of which are familiar to many. The spiciness of chili peppers from capsaicin action at TRPV1, the pungency of garlic and wasabi produced by allicin and isothiocyanate modulation of TRPA1 (Jordt et al., 2004; Macpherson et al., 2005), and the cool, minty sensation of toothpastes or candies brought upon by menthol modulation of TRPM8 (Moran, 2018) are sought after as welcomed, albeit sometimes discomforting, sensations. However, in the case of chronic pain conditions, whether due to nerve injury or inflammation, ongoing painful stimulation leads to peripheral and central sensitization that can lead to painful sensations upon mild tactile stimulation (allodynia), greater than normal pain response to a stimulus (hyperalgesia), and spontaneous pain (Walker and Huang, 2002; Patapoutian et al., 2009).

\section{DIFFERENCES IN THE PUTATIVE CBD BINDING SITE OF THE IONOTROPIC CANNABINOID RECEPTORS}

Recently, a cryo-EM structure of rTRPV2 interacting with CBD has been elucidated in two separate states (Pumroy et al., 2019). The putative binding location of CBD was identified as the region between helix 6 of one monomer and helix 5 of the adjacent monomer (see Figure 2A), a general schematic of which can be found in the Supplementary Material. By using the information provided from the cryo-EM structures, we can identify regions of sequential homology and structural similarity in the putative CBD binding site across the ionotropic cannabinoid receptors. The sequence alignment (Figure 1) details the similarities between rTRPV2 and hTRPV2, as well as the human sequence of other ionotropic cannabinoid receptors TRPV1, TRPV3, TRPV4, TRPA1, and TRPM8. To get an idea of the binding site, residues within $10 \AA$ of the putative CBD binding location in rTRPV2 these have been highlighted for analysis and directly compared to the other ionotropic cannabinoid receptors. A visual representation of the putative binding site(s) in three ionotropic cannabinoid receptors can be seen in Figures 2A-C.

\section{TRPV1}

One of the most well-studied TRP channels is TRPV1, which shares an overall sequence identity of 50\% with hTRPV2 (Bishnoi and Premkumar, 2013). TRPV1 is primarily known for its activation via vanilloid agonists, like capsaicin, and the subsequent desensitization it undergoes leading to the 


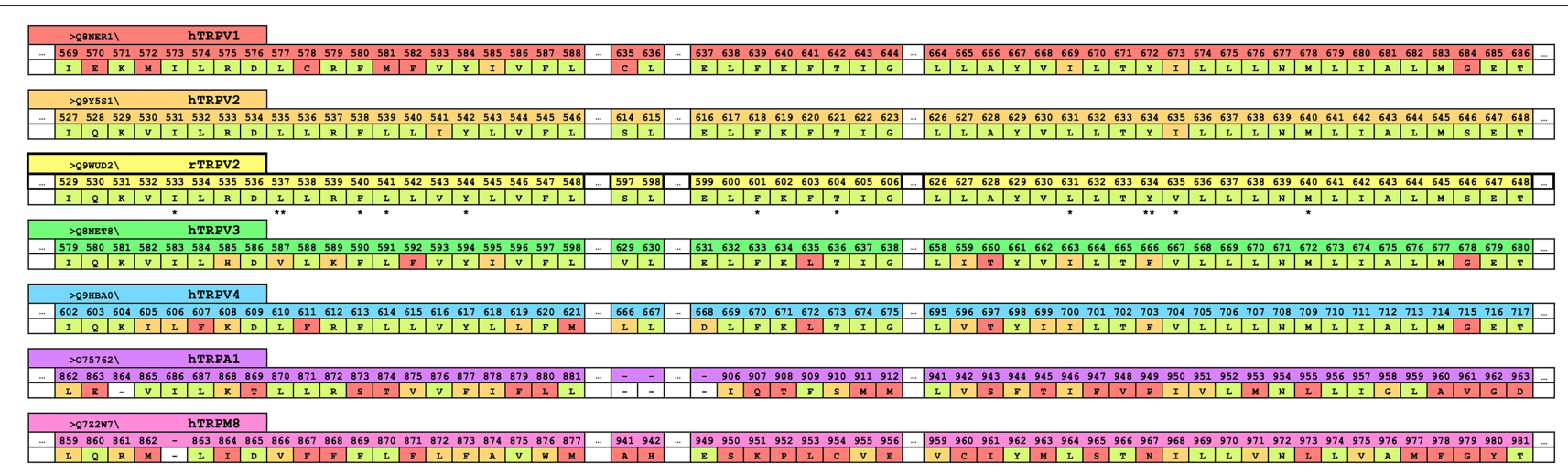

FIGURE 1 | A truncated version of the human sequence alignment of six ionotropic cannabinoid receptors and rTRPV2. CBD has been resolved in rTRPV2 in two separate states and residues within $10 \AA$ of the putative binding site of CBD have been highlighted and are shown here as a reference (yellow row). Comparable regions within the human ionotropic cannabinoid receptors have been aligned. Residues within hTRPV1 (red row), hTRPV2 (orange row), hTRPV3 (green row), hTRPV4 (blue row), hTRPA1 (purple row), and hTRPM8 (pink row) that are the same as the reference (rTRPV2) are shown in pale green. Residues that are of a similar type to the reference are shown in orange, and divergent residues are shown in red. The double starred residues, L537 and Y634, indicate the two residues that were noted to have rotameric changes from the apo to the CBD-bound structure of rTRPV2 and are visualized in Figure 2 . Single starred residues are within $5 \AA$ of bound $\mathrm{CBD}$ and are marked for easy vertical comparison across the ionotropic cannabinoid receptors.

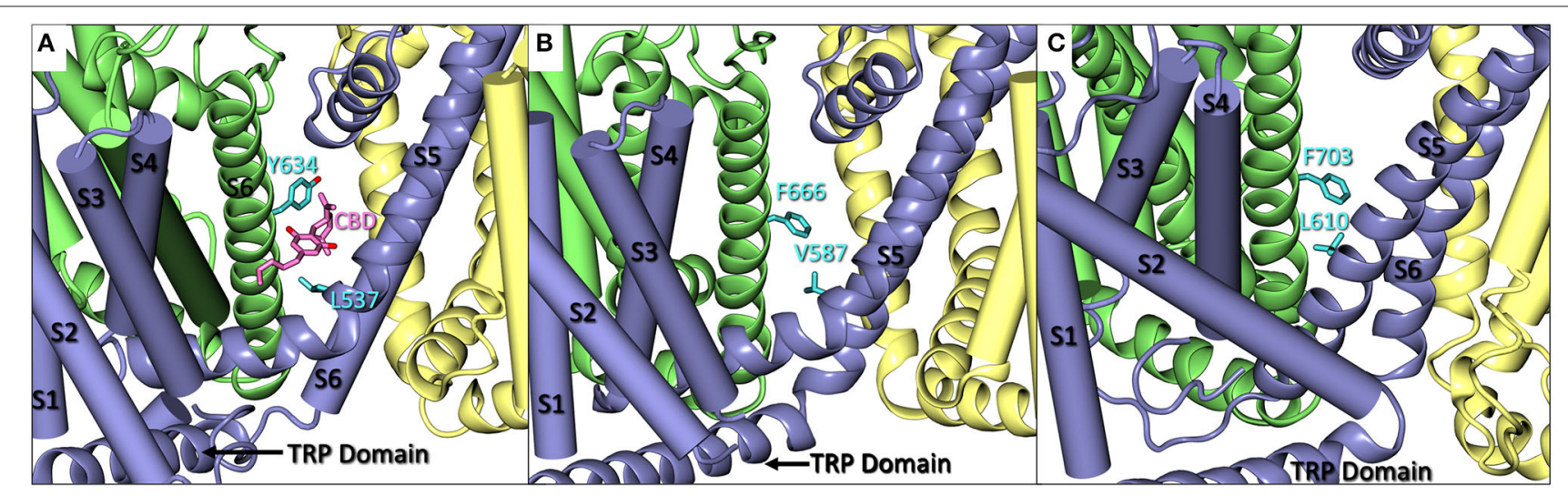

FIGURE 2 | (A) A close up of CBD (pink) bound in rTRPV2 (adapted from PDB: 6U88) with Y634 and L537 in cyan. Helices S1-S4 and S6 are shown as cartoon tubes with S5, the pore helix, and the TRP domain shown as cartoon ribbons. (B) A close up of hTRPV3 (adapted from PDB: 6MHO) with comparable residues F666 and V587 shown in cyan. Helices S1-S4 are shown as cartoon tubes and S5-TRP domain are shown as cartoon ribbons. (C) A close up of Xenopus tropicalis TRPV4 (adapted from PDB: 6BBJ) which shares 78\% sequence homology with human TRPV4. F703 and L610 are shown in cyan. Helices S1-S4 are shown as cartoon tubes, and S5-TRP domain are shown as cartoon ribbons.

paradoxical analgesic effect elicited (Wong and Gavva, 2009). This effect is sought after for its potential use in pain therapies and is the basis of how some current topical-based therapies work, like Capzasin cream. TRPV1 shares the highest sequence identity within the putative $\mathrm{CBD}$ binding site as described above at $79 \%$, second only to TRPV2 itself. Additionally, CBD is reported to have the highest efficacy of the vanilloid subfamily at TRPV1 ( 78\%) (Chianese et al., 2020).

Two residues that have been identified for their involvement of CBD binding in TRPV2, L535, and Y634 (Pumroy et al., 2019), can also be found in TRPV1 as L577 and Y672. L577 and Y672 are in comparable sequential and physical locations to their TRPV2 counterpart in addition to fairly consistent sequence homology about the rest of the binding site as seen in Figure 1.
However, the tyrosine in either channel is unlikely to have a direct interaction with $\mathrm{CBD}$ in the putative binding site. Assuming the CBD binding site in TRPV1 is the same as that of TRPV2, this orientation of tyrosine would likely have minimal impact in the binding affinity of $\mathrm{CBD}$, especially due to the reported role of Y634 in TRPV2 as a hydrophobic shield from water in the pore. Due to this, it is possible that Y672 in TRPV1 is still providing some degree of hydrophobic shielding for CBD should it bind in the same location, while the lipophilic interactions via L577 and surrounding lipophilic residues remain intact.

\section{TRPV2}

TRPV2 is the thermoTRP with the highest temperature activation threshold among its subgroup with activation occurring above 
$52^{\circ} \mathrm{C}$ (Caterina et al., 1999). While TRPV2 is insensitive to capsaicin, it undergoes similar desensitization following activation and is deeply involved in inflammation and chronic pain (Levine and Alessandri-Haber, 2007). The cryo-EM structure of CBD in rTRPV2 was published in 2019 by Pumroy et al. and this structure allows us to delve deeper into how CBD interacts with TRPV2 specifically (Pumroy et al., 2019), but also hypothesize on the binding of CBD in other TRP channels that can be modulated by this cannabinoid ligand via sequential comparison and computational exploration.

A comparison of the rTRPV2 and hTRPV2 putative binding site of CBD shows $96 \%$ sequence homology with few instances of changes between hydrophobic residues, such as valine (rTRPV2) to isoleucine (hTRPV2). Beyond this, the polarity and aromaticity of residues within the binding site between rTRPV2 and hTRPV2 remains consistent (see Figure 1). Rotameric comparisons between the apo (PDB: 6U84 and 6U86) and CBDbound (PDB: 6U8A and 6U88) cryo-EM structures for rTRPV2 revealed several rotameric changes throughout. Though most rotameric changes were peripheral and not within the defined scope of the CBD binding site, two rotameric changes were located within this scope. In the apo structure, Y634 takes on a $\mathrm{g}+$ conformation while the CBD-bound structure shows Y634 adapting a trans conformation (Figure 2A). This movement shifts the hydroxyl group of the tyrosine toward the pore, believed to create some hydrophobic shielding for $\mathrm{CBD}$ from ions and water found there (Pumroy et al., 2019). Additionally, L537 shows a rotameric change, going from trans in the apo structure to $\mathrm{g}+$ in the CBD-bound structure which is reported to allow accommodation for the aromatic ring of CBD. With two CBDbound cryo-EM structures identifying CBD in the same general location (Pumroy et al., 2019) and a reported efficacy of $\sim 67 \%$ at TRPV2 (Chianese et al., 2020), exploring this region should provide insight on the binding mode of CBD.

\section{TRPV3}

TRPV3 is predominantly expressed in the brain (Xu et al., 2002) as well as several peripheral tissues like the skin and tongue (Wang et al., 2011). Additionally, TRPV3 acts as a thermosensor for innocuous warm temperatures, activating between 33 and $39^{\circ} \mathrm{C}$ (Szallasi et al., 2007). During a screen with a variety of phytocannabinoids, CBD was observed to have a potency similar to that of its typical agonist (De Petrocellis et al., 2012), carvacrol, though the efficacy of CBD at TRPV3 is poorer than that of TRPV1 and TRPV2 ( $\sim 54$ vs. $\sim 78, \sim 67 \%$, respectively). With $77 \%$ sequence homology in the CBD putative binding site, some small differences in sequence might be responsible for the low efficacy.

In TRPV2, Y634 is said to provide hydrophobic shielding for CBD by shifting to point the polar hydroxyl group toward the pore of the channel, preventing solvation of the location where CBD is proposed to bind. In TRPV3, this tyrosine is replaced by F666, losing the polar hydroxyl group which could lessen the shielding effect, but also occlude the binding site due to not being able to twist and point to the pore. Another sequential change that could impact the efficacy of CBD at TRPV3 is the change of leucine (L537, TRPV2) to valine (V587, TRPV3). While both hydrophobic, the shorter chain of the valine might affect the extent of the lipophilic interactions within TRPV3 (Figure 2B). While the efficacy of CBD at TRPV3 is generally considered poor, it still retains a submicromolar potency of around $0.51 \mu \mathrm{M}$ (Chianese et al., 2020).

\section{TRPV4}

The final vanilloid subfamily member discussed in this perspective is TRPV4. Similar to TRPV3, TRPV4 responds to warm thermal changes of temperatures ranging from 25 to $34^{\circ} \mathrm{C}$ (Vay et al., 2012). Additionally, this channel, located in cutaneous A and C fibers (Suzuki et al., 2003), plays a role in skin barrier function and nociception (Nilius and Owsianik, 2010).

Initially, it appears that there are only moderate differences in the putative CBD binding site in TRPV4 and TRPV2 due to the $68 \%$ sequence homology of this region. However, CBD is the least efficacious at TRPV4 with a mere 15\% efficacy (Chianese et al., 2020). The aspect that likely has the largest effect on CBD binding is the structurally different helical arrangement that TRPV4 takes in comparison to its family members. TRP channels in this subgroup typically adopt a "straddling" formation of S1-S4 over the TRP domain where S1 and S4 reside on one side of the TRP domain and S2 and S3 reside on the other. TRPV4, however, does not appear to follow suit. In a series of recently published cryoEM structures of Xenopus tropicalis TRPV4, which maintains 78\% sequence homology with hTRPV4 (Deng et al., 2018), helical packing of S1-S4 against S5-S6 was observed to be different from that of other resolved TRPV channels, such as TRPV1, TRPV2, and TRPV3 (Deng et al., 2018). Helices S1, S3, and S4 all appear on one side of the TRP domain, leaving S2 on the other. This differentiation in helical arrangement, as well as the angle at which S2 takes on in TRPV4, alters the shape of the S1-S4 bundle (see Figure 2C), affecting the putative CBD binding site by altering the interaction between S5 and S6 of adjacent monomers. Deng et al. note that this unique $\mathrm{S} 1-\mathrm{S} 4$ packing arrangement may be due to truncation of TRPV4, though it is reported that only the unstructured $\mathrm{N}$ - and C-termini were truncated. Additionally, multiple TRPV4 structures with various cations were resolved, all maintaining this feature. The S4 helix obstructs the putative CBD binding site located in this channel, lending support that this strange arrangement of S1-S4 helices is plausible rather than an artifact of truncation and/or crystallization.

\section{TRPA1}

The first member of the ankyrin subfamily, TRPA1, can be found co-expressed with TRPV1 in a subset of peripheral sensory neurons (Patapoutian et al., 2009). TRPA1 is activated by isothiocyanates, pungent compounds found in mustard, garlic, and onions (Niforatos et al., 2007), covalently binding to an internal cysteine or lysine residue located on its extensive ankyrin repeat domain. With regard to its role as a thermoTRP, TRPA1 can be found on the lowest end of the temperature scale, activating below temperatures of $17^{\circ} \mathrm{C}$ (Story et al., 2003). Additionally, TRPA1 plays an important role in neuropathic and inflammatory pain through the mediation of bradykinin-evoked and mechanical hyperalgesia (Jordt et al., 2004). 
CBD has been shown to act as an agonist at TRPA1 with an efficacy of $108 \%$ compared to its usual agonist of allylisothiocyanate $(100 \mu \mathrm{M})$ (Chianese et al., 2020), and while the putative $\mathrm{CBD}$ binding site is structurally comparable to that of the TRPVs, there is low sequence homology within the binding site $(\sim 30 \%)$. Similar to the previous TRP channels discussed, TRPA1 maintains a leucine in the same position as in TRPV2 (L870 and L537, respectively). Looking at the sequence alignment (Figure 1), hydrophobic residues are readily present and are, in fact, pointing in the region where $\mathrm{CBD}$ is proposed to bind. However, since the efficacy of CBD at TRPA1 is considerably higher than TRPV2, the sequence homology with TRPV2 might be of little importance in this case.

\section{TRPM8}

Finally, the last member of the ionotropic cannabinoid receptors that will be discussed here is TRPM8. TRPM8 is activated by temperatures below $27^{\circ} \mathrm{C}$ (de la Peña et al., 2005) as well as by compounds that elicit a "cooling" effect, such as menthol, eucalyptol, and icilin (McKemy et al., 2002; Peier et al., 2002b; Chuang et al., 2004). Compounds tested at TRPM8 are usually tested for their antagonism against both menthol and icilin as they are reported to activate the channel in slightly different locations (Chuang et al., 2004; Bandell et al., 2006; Yin et al., 2018; $\mathrm{Xu}$ et al., 2020). For both ligands, CBD acts as an antagonist at submicromolar concentrations (De Petrocellis et al., 2008).

Because of this, it is slightly more difficult to hypothesize if CBD binds in the same location in TRPM8 as it would in the TRPV or TRPA subfamilies. The binding site in TRPM8 has $\sim 30 \%$ sequence homology with that of TRPV2, but because $\mathrm{CBD}$ acts as an antagonist, the location of binding as well as the movements required by the channel to become inactive, could require different mechanisms than activation of the other ionotropic cannabinoid receptors.

\section{CONCLUDING REMARKS AND FUTURE DIRECTIONS}

Targeting the endocannabinoid system has been a promising strategy for the modulation of pain (Maione et al., 2006; Lau and Vaughan, 2014; Aizpurua-Olaizola et al., 2017; Chanda et al., 2019). One in particular, CBD, has gained mainstream attention in recent years due to over the counter (OTC) uses in balms, creams, tinctures, and more for joint and muscle pain, neuroprotection, anti-nausea, anti-inflammation, and anxiolytic properties (Mechoulam et al., 2007), as well as pharmaceutical uses in drugs, such as Sativex, a 1:1 CBD:THC oromucosal spray has been approved for use in the UK to aid in the relief of multiple sclerosis (MS) related symptoms, and Epidiolex, an FDA-approved CBD-based drug used to treat two severe forms of pediatric epilepsy. Additionally, there is literature precedent to support the claims of $\mathrm{CBD}$ as a means to treat other conditions, such as arthritis (Malfait et al., 2000), anxiety (Guimaraes et al., 1994), and the potential for treatment in substance use disorders (Wiese and WilsonPoe, 2018; Turna et al., 2019). By seeking to understand the targets of cannabinoid ligands, and particularly where they bind, researchers will be better equipped to design drugs to treat chronic pain disorders.

Recently, a dimerized version of CBD (CBDD or cannabitwinol) has been isolated, structurally characterized, and tested by Chianese et al. at the ionotropic cannabinoid receptors (Chianese et al., 2020). What was discovered was that CBDD was found to be nearly inactive at TRPV1 and TRPV2, in contrast to the good efficacies of CBD at these two channels, while exhibiting poor efficacy at TRPV3 and TRPV4, and retaining activity as a TRPA1 activator ( $\sim 97 \%$ efficacy) and TRPM8 inhibitor against icilin $\left(\mathrm{IC}_{50}=3.9 \pm 0.4 \mu \mathrm{M}\right)$. One structural factor that may play a role in how well CBDD interacts with TRPA1, despite its doubling in size, is the TRP-like domain which lies lower in the intracellular region than a TRP domain (Paulsen et al., 2015). The TRP domain found in TRPV1-4 and TRPM8 is nestled just below the lower leaflet in the intracellular region of the cell. In TRPV2, the S1-S4 domain straddles the TRP domain which acts as a "floor" to the putative CBD binding site. Since this feature is not present in TRPA1, instead having a TRP-like domain, more space is created in the putative binding region, potentially allowing for easier access by CBD and its dimerized sibling. If we hypothesize that CBDD binds in the same location as CBD in TRPA1, it is sensible to think that the increase in room from the TRP-like domain would allow more space for CBDD to fit, even if one-half of dimerized CBD were to bind, potentially leaving the other half "left out" of the binding site. Conversely, based on the features discussed here, CBDD would have limited space in TRPV1-TRPV4.

The comparison of the putative CBD binding site presented here is based on the sequence alignment of the ionotropic cannabinoid receptors and previously published crystal or cryoEM structures from a molecular modeling perspective. Ideally, future work would combine cryo-EM/crystal structures of CBD interacting with the other TRP channels in addition to sitedirected mutagenesis to further investigate these interactions.

\section{DATA AVAILABILITY STATEMENT}

The original contributions presented in the study are included in the article/Supplementary Material, further inquiries can be directed to the corresponding author/s.

\section{AUTHOR CONTRIBUTIONS}

$\mathrm{CM}$ wrote the manuscript and prepared the figures with guidance and editing from PR. All authors contributed to the article and approved the submitted version.

\section{FUNDING}

This work was supported by the National Institute on Drug Abuse grant KO5 (DA021358) to PR. 


\section{ACKNOWLEDGMENTS}

We wish to acknowledge Paula Morales for her feedback and insights that helped aid in the conception of this perspective article.

\section{REFERENCES}

Aizpurua-Olaizola, O., Elezgarai, I., Rico-Barrio, I., Zarandona, I., Etxebarria, N., and Usobiaga, A. (2017). Targeting the endocannabinoid system: future therapeutic strategies. Drug Discov. Today 22, 105-110. doi: 10.1016/j.drudis.2016.08.005

Akopian, A. N., Ruparel, N. B., Jeske, N. A., Patwardhan, A., and Hargreaves, K. M. (2009). Role of ionotropic cannabinoid receptors in peripheral antinociception and antihyperalgesia. Trends Pharmacol. Sci. 30, 79-84. doi: 10.1016/j.tips.2008.10.008

Bandell, M., Dubin, A. E., Petrus, M. J., Orth, A., Mathur, J., Sun, W. H., et al. (2006). High-throughput random mutagenesis screen reveals TRPM8 residues specifically required for activation by menthol. Nat. Neurosci. 9, 493-500. doi: $10.1038 / \mathrm{nn} 1665$

Bautista, D. M., Movahed, P., Hinman, A., Axelsson, H. E., Sterner, O., Högestätt, E. D., et al. (2005). Pungent products from garlic activate the sensory ion channel TRPA1. Proc. Natl. Acad. Sci. U.S.A. 102, 12248-12252. doi: 10.1073/pnas.0505356102

Bender, F. L., Mederos Schnitzler, M., Li, Y., Ji, A., Weihe, E., Gudermann, T., et al. (2005). The temperature-sensitive ion channel tRPV2 is endogenously expressed and functional in the primary sensory cell line F-11. Cell. Physiol. Biochem. 15, 183-194. doi: 10.1159/000083651

Bishnoi, M., and Premkumar, L. S. (2013). Changes in TRP channels expression in painful conditions. Open Pain J. 6, 10-22. doi: 10.2174/1876386301306010010

Caterina, M. J. (2006). Transient receptor potential ion channels as participants in thermosensation and thermoregulation. Am. J. Physiol. Regul. Integr. Comp. Physiol. 292, R64-R76. doi: 10.1152/ajpregu.00446.2006

Caterina, M. J. (2014). TRP channel cannabinoid receptors in skin sensation, homeostasis, and inflammation. ACS Chem. Neurosci. 5, 1107-1116. doi: $10.1021 / \mathrm{cn} 5000919$

Caterina, M. J., Rosen, T. A., Tominaga, M., Brake, A. J., and Julius, D. (1999). A capsaicin-receptor homologue with a high threshold for noxious heat. Nature 398, 436-441. doi: 10.1038/18906

Caterina, M. J., Schumacher, M. A., Tominaga, M., Rosen, T. A., Levine, J. D., and Julius, D. (1997). The capsaicin receptor: a heat-activated ion channel in the pain pathway. Nature 389, 816-824. doi: 10.1038/39807

Chanda, D., Neumann, D., and Glatz, J. F. C. (2019). The endocannabinoid system: overview of an emerging multi-faceted therapeutic target. Prostag. Leukotr. Ess. Fatty Acids 140, 51-56. doi: 10.1016/j.plefa.2018.11.016

Chianese, G., Lopatriello, A., Schiano-Moriello, A., Caprioglio, D., Mattoteia, D., Benetti, E., et al. (2020). Cannabitwinol, a dimeric phytocannabinoid from hemp, Cannabis sativa L., is a selective thermo-TRP modulator. J. Nat. Prod. 83, 2727-2736. doi: 10.1021/acs.jnatprod.0c00668

Chuang, H. H., Neuhausser, W. M., and Julius, D. (2004). The super-cooling agent icilin reveals a mechanism of coincidence detection by a temperature-sensitive TRP channel. Neuron 43, 859-869. doi: 10.1016/j.neuron.2004.08.038

de la Peña, E., Mälkiä, A., Cabedo, H., Belmonte, C., and Viana, F. (2005). The contribution of TRPM8 channels to cold sensing in mammalian neurones. J. Physiol. 567, 415-426. doi: 10.1113/jphysiol.2005.086546

De Petrocellis, L., Orlando, P., Moriello, A. S., Aviello, G., Stott, C., Izzo, A. A., et al. (2012). Cannabinoid actions at TRPV channels: effects on TRPV3 and TRPV4 and their potential relevance to gastrointestinal inflammation. Acta Physiol. 204, 255-266. doi: 10.1111/j.1748-1716.2011.02338.x

De Petrocellis, L., Vellani, V., Schiano-Moriello, A., Marini, P., Magherini, P. C., Orlando, P., et al. (2008). Plant-derived cannabinoids modulate the activity of transient receptor potential channels of ankyrin type-1 and melastatin type-8. J. Pharmacol. Exp. Ther. 325, 1007-1015. doi: 10.1124/jpet.107.134809

Deng, Z., Paknejad, N., Maksaev, G., Sala-Rabanal, M., Nichols, C. G., Hite, R. K., et al. (2018). Cryo-EM and X-ray structures of TRPV4 reveal insight into

\section{SUPPLEMENTARY MATERIAL}

The Supplementary Material for this article can be found online at: https://www.frontiersin.org/articles/10.3389/fncel. 2020.615811/full\#supplementary-material

ion permeation and gating mechanisms. Nat. Struct. Mol. Biol. 25, 252-260. doi: 10.1038/s41594-018-0037-5

Guimaraes, F. S., de Aguiar, J. C., Mechoulam, R., and Breuer, A. (1994) Anxiolytic effect of cannabidiol derivatives in the. Gen. Pharmacol. 25, 161-164. doi: 10.1016/0306-3623(94)90027-2

Howlett, A. C. (2002). The cannabinoid receptors. Prostag. Other Lipid Mediat. 68-69, 619-631. doi: 10.1016/S0090-6980(02)00060-6

Ilgen, M. A., Bohnert, K., Kleinberg, F., Jannausch, M., Bohnert, A. S. B., Walton, M., et al. (2013). Characteristics of adults seeking medical marijuana certification. Drug Alcohol Depend. 132, 654-659. doi: 10.1016/j.drugalcdep.2013.04.019

Jordt, S. E., Bautista, D. M., Chuang, H. H., McKemy, D. D., Zygmunt, P. M., Högestätt, E. D., et al. (2004). Mustard oils and cannabinoids excite sensory nerve fibres through the TRP channel ANKTM1. Nature 427, 260-265. doi: $10.1038 /$ nature02282

Klimkiewicz, A., and Jasinska, A. (2018). The health effects of cannabis and cannabinoids. Psychiatria 15, 88-92. doi: 10.17226/24625

Kobayashi, K., Fukuoka, T., Obata, K., Yamanaka, H., Dai, Y., Tokunaga, A., et al. (2005). Distinct expression of TRPM8, TRPA1, and TRPV1 mRNAs in rat primary afferent neurons with A $/ \mathrm{C}$-fibers and colocalization with TRK receptors. J. Comp. Neurol. 493, 596-606. doi: 10.1002/cne.20794

Lau, B. K., and Vaughan, C. W. (2014). Targeting the endogenous cannabinoid system to treat neuropathic pain. Front. Pharmacol. 5:28. doi: 10.3389/fphar.2014.00028

Levine, J. D., and Alessandri-Haber, N. (2007). TRP channels: targets for the relief of pain. Biochim. Biophys. Acta Mol. Basis Dis. 1772, 989-1003. doi: 10.1016/j.bbadis.2007.01.008

Luongo, L., Starowicz, K., Maione, S., and Di Marzo, V. (2017). Allodynia lowering induced by cannabinoids and endocannabinoids (ALICE). Pharmacol. Res. 119, 272-277. doi: 10.1016/j.phrs.2017.02.019

Macpherson, L. J., Geierstanger, B. H., Viswanath, V., Bandell, M., Eid, S. R., Hwang, S. W., et al. (2005). The pungency of garlic: activation of TRPA1 and TRPV1 in response to allicin. Curr. Biol. 15, 929-934. doi: 10.1016/j.cub.2005.04.018

Maione, S., Starowicz, K., Palazzo, E., Rossi, F., and Di Marzo, V. (2006). The endocannabinoid and endovanilloid systems and their interactions in neuropathic pain. Drug Dev. Res 67, 339-354. doi: 10.1002/ddr.20098

Malfait, A. M., Gallily, R., Sumariwalla, P. F., Malik, A. S., Andreakos, E., Mechoulam, R., et al. (2000). The nonpsychoactive cannabis constituent cannabidiol is an oral anti-arthritic therapeutic in murine collagen-induced arthritis. Proc. Natl. Acad. Sci. U.S.A. 97, 9561-9566. doi: 10.1073/pnas.160105897

Mandadi, S., Sokabe, T., Shibasaki, K., Katanosaka, K., Mizuno, A., Moqrich, A., et al. (2009). TRPV3 in keratinocytes transmits temperature information to sensory neurons via ATP. Pflugers Arch. Eur. J. Physiol. 458, 1093-1102. doi: 10.1007/s00424-009-0703-x

McKemy, D. D., Neuhausser, W. M., and Julius, D. (2002). Identification of a cold receptor reveals a general role for TRP channels in thermosensation. Nature 416, 52-58. doi: 10.1038/nature719

Mechoulam, R., Peters, M., Murillo-Rodriguez, E., and Hanuš, L. O. (2007). Cannabidiol-recent advances. Chem. Biodiv. 4, 1678-1692. doi: 10.1002/cbdv.200790147

Morales, P., Isawi, I., and Reggio, P. H. (2018). Towards a better understanding of the cannabinoid-related orphan receptors GPR3, GPR6, and GPR12. Drug Metab. Rev. 50, 74-93. doi: 10.1080/03602532.2018.14 28616

Moran, M. M. (2018). TRP channels as potential drug targets. Annu. Rev. Pharmacol. Toxicol. 58, 309-330. doi: 10.1146/annurev-pharmtox-010617-052832 
Muller, C., Morales, P., and Reggio, P. H. (2019). Cannabinoid ligands targeting TRP channels. Front. Mol. Neurosci. 11:487. doi: 10.3389/fnmol.2018.00487

Nielsen, S., Sabioni, P., Trigo, J. M., Ware, M. A., Betz-Stablein, B. D., Murnion, B., et al. (2017). Opioid-sparing effect of cannabinoids: a systematic review and meta-analysis. Neuropsychopharmacology 42, 1752-1765. doi: 10.1038/npp.2017.51

Niforatos, W., Zhang, X., Lake, M. R., Walter, K., a, Neelands, T., Holzman, T. F., et al. (2007). Activation of TRPAl channels by the fatty acid amide hydrolase inhibitor $3 \mathrm{~J}$-carbamoylbiphenyl-3-yl. Mol. Pharmacol. 71, 1209-1216. doi: 10.1124/mol.106.033621

Nilius, B., and Owsianik, G. (2010). Transient receptor potential channelopathies. Pflugers Arch. Eur. J. Physiol. 460, 437-450. doi: 10.1007/s00424-010-0788-2

Nilius, B., and Vennekens, R. (2007). TRP channels and human diseases. Biochem. Biophys. Acta 1772, 805-812. doi: 10.1016/j.bbadis.2007.02.002

Patapoutian, A., Tate, S., and Woolf, C. J. (2009). Transient receptor potential channels: targeting pain at the source. Nat. Rev. Drug Discov. 8, 55-68. doi: $10.1038 / \mathrm{nrd} 2757$

Paulsen, C. E., Armache, J. P., Gao, Y., Cheng, Y., and Julius, D. (2015). Structure of the TRPA1 ion channel suggests regulatory mechanisms. Nature 520, 511-517. doi: $10.1038 /$ nature 14367

Peier, A. M., Moqrich, A., Hergarden, A. C., Reeve, A. J., Andersson, D. A., Story, G. M., et al. (2002b). A TRP channel that senses cold stimuli and menthol. Cell 108, 705-715. doi: 10.1016/S0092-8674(02)00652-9

Peier, A. M., Reeve, A. J., Andersson, D. A., and Moqrich, A. (2002a). A heatsensitive TRP channel expressed in keratinocytes. Science 296, 2046-2049. doi: $10.1126 /$ science. 1073140

Perálvarez-Marín, A., Doñate-Macian, P., and Gaudet, R. (2013). What do we know about the transient receptor potential vanilloid 2 (TRPV2) ion channel? FEBS J. 280, 5471-5487. doi: 10.1111/febs.12302

Pertwee, R. G., Howlett, A. C., Abood, M. E., Alexander, S. P. H., Di Marzo, V., Elphick, M. R., et al. (2010). International union of basic and clinical pharmacology. LXXIX. Cannabinoid receptors and their ligands: beyond CB1 and CB2. Pharmacol. Rev. 62, 588-631. doi: 10.1124/pr.110.003004

Pumroy, R. A., Samanta, A., Liu, Y., Hughes, T. E. T., Zhao, S., Yudin, Y., et al. (2019). Molecular mechanism of TRPV2 channel modulation by cannabidiol. bioRxiv 521880. doi: 10.7554/eLife.48792

Sanchez, J. F., Krause, J. E., and Cortright, D. N. (2001). The distribution and regulation of vanilloid receptor VR1 and VR1 5P splice variant rna expression in rat. Neuroscience 107, 373-381. doi: 10.1016/s0306-4522(01)00373-6 Available online at: www.neuroscience-ibro.com.

Starkus, J., Jansen, C., Shimoda, L. M. N., Stokes, A. J., Small-Howard, A. L., and Turner, H. (2019). Diverse TRPV1 responses to cannabinoids. Channels 13, 172-191. doi: 10.1080/19336950.2019.1619436

Story, G. M., Peier, A. M., Reeve, A. J., Eid, S. R., Mosbacher, J., Hricik, T. R., et al. (2003). ANKTM1, a TRP-like channel expressed in nociceptive neurons, is activated by cold temperatures. Cell 112, 819-829. doi: 10.1016/S0092-8674(03)00158-2

Suzuki, M., Watanabe, Y., Oyama, Y., Mizuno, A., Kusano, E., Hirao, A., et al. (2003). Localization of mechanosensitive channel TRPV4 in mouse skin. Neurosci. Lett. 353, 189-192. doi: 10.1016/j.neulet.2003.09.041
Szallasi, A., Cortright, D. N., Blum, C. A., and Eid, S. R. (2007). The vanilloid receptor TRPV1: 10 years from channel cloning to antagonist proof-of-concept. Nat. Rev. Drug Discov. 6, 357-372. doi: 10.1038/nrd2280

Tominaga, M., Caterina, M. J., Malmberg, A. B., Rosen, T. A., Gilbert, H., and Skinner, K. (1998). The cloned capsaicin receptor integrates multiple painproducing stimuli. Neuron 21, 531-543. doi: 10.1016/S0896-6273(00)80564-4

Turna, J., MacKillop, J., Rush, B., Frey, B. N., Syan, S. K., Costello, J., et al. (2019). Cannabidiol as a novel candidate alcohol use disorder pharmacotherapy: a systematic review. Alcoholism 55, 1-14. doi: 10.1111/acer.13964

Vay, L., Gu, C., and McNaughton, P. A. (2012). The thermo-TRP ion channel family: properties and therapeutic implications. Br. J. Pharmacol. 165, 787-801. doi: 10.1111/j.1476-5381.2011.01601.x

Walker, J. M., and Huang, S. M. (2002). Cannabinoid analgesia. Pharmacol. Ther. 95, 127-135. doi: 10.1016/s0163-7258(02)00252-8

Wang, B., Danjo, A., Kajiya, H., Okabe, K., and Kido, M. A. (2011). Oral epithelial cells are activated via TRP channels. J. Dent. Res. 90, 163-167. doi: 10.1177/0022034510385459

Ware, M. A., Mrcp, M., Gamsa Phd, A., Persson, J., Fitzcharles, M.-A., Ware, M. A., et al. (2002). Cannabis for chronic pain: case series and implications for clinicians. Pain Res. Manag. 7, 95-99. doi: 10.1155/2002/380509

Wiese, B., and Wilson-Poe, A. R. (2018). Emerging evidence for cannabis role in opioid use disorder. Cannabis Cannabinoid Res. 3, 179-189. doi: $10.1089 /$ can.2018.0022

Winter, Z., Buhala, A., Ötvös, F., Jósvay, K., Vizler, C., Dombi, G., et al. (2013). Functionally important amino acid residues in the transient receptor potential vanilloid 1 (TRPV1) ion channel-an overview of the current mutational data. Mol. Pain 9:30. doi: 10.1186/1744-8069-9-30

Wong, G. Y., and Gavva, N. R. (2009). Therapeutic potential of vanilloid receptor TRPV1 agonists and antagonists as analgesics: recent advances and setbacks. Brain Res. Rev. 60, 267-277. doi: 10.1016/j.brainresrev.2008.12.006

Xu, H., Ramsey, I. S., Kotecha, S. A., Moran, M. M., Chong, J. A., Lawson, D., et al. (2002). TRPV3 is a calcium-permeable temperature-sensitive cation channel. Nature 418, 181-186. doi: 10.1038/nature00882

Xu, L., Han, Y., Chen, X., Aierken, A., Wen, H., Zheng, W., et al. (2020). Molecular mechanisms underlying menthol binding and activation of TRPM8 ion channel. Nat. Commun. 11:3790. doi: 10.1016/j.bpj.2019.11.2337

Yin, Y., Wu, M., Zubcevic, L., Borschel, W. F., Lander, G. C., and Lee, S. Y. (2018). Structure of the cold- and menthol-sensing ion channel TRPM8. Science 359, 237-241. doi: 10.1126/science.aan 4325

Conflict of Interest: The authors declare that the research was conducted in the absence of any commercial or financial relationships that could be construed as a potential conflict of interest.

Copyright (C) 2020 Muller and Reggio. This is an open-access article distributed under the terms of the Creative Commons Attribution License (CC BY). The use, distribution or reproduction in other forums is permitted, provided the original author(s) and the copyright owner(s) are credited and that the original publication in this journal is cited, in accordance with accepted academic practice. No use, distribution or reproduction is permitted which does not comply with these terms. 